\title{
YOU CAN SEE THE ARROWS IN A QUIVER OPERATOR ALGEBRA
}

\section{BARUCH SOLEL}

(Received 19 September 2002; revised 23 April 2003)

Communicated by G. Wills

\begin{abstract}
We prove that two quiver operator algebras can be isometrically isomorphic only if the quivers (= directed graphs) are isomorphic. We also show how the graph can be recovered from certain representations of the algebra.
\end{abstract}

2000 Mathematics subject classification: primary 47L55, 47L75, 46K50.

Keywords and phrases: $C^{*}$-correspondence, tensor algebra, quiver algebra, free semigroup algebra, free semigroupoid algebra, isomorphism, directed graph.

\section{Introduction}

A quiver is a directed graph with $\mathrm{n}$ vertices $\{1,2, \ldots, n\}$ and $C_{i j}$ arrows from $j$ to $i$. Here $C_{i j}$ is a non negative integer (or possibly $\infty$ if the graph is infinite). Let $A$ be the $C^{*}$-direct sum of $n$ copies of $\mathbb{C}$ indexed by the vertices. For a finite graph we shall view $A$ as the algebra $D_{n}$ of all diagonal $n \times n$ matrices.

As we explain in the next section, one can associate with the quiver a correspondence $E(C)$ over $A$ and this correspondence gives rise to a (non selfadjoint) operator algebra, denoted $\mathscr{T}_{+}(C)$, that is referred to as the quiver algebra. Another algebra associated with the quiver is $H^{\infty}(C)$, the $w^{*}$-closure of $\mathscr{T}_{+}(C)$. (Here $C$ is the $n \times n$ matrix $\left\{C_{i j}\right\}$ associated with the quiver). In fact, $\mathscr{T}_{+}(C)$ is the tensor algebra associated with the correspondence $E(C)$. (See [12] for more about tensor algebras and their relation to Cuntz-Pimsner algebras).

If $C$ is the $1 \times 1$ matrix whose entry is $n$, we have $E(C)=\mathbb{C}^{n}$ and the quiver algebra $\mathscr{T}_{+}(C)$ is the non commutative disc algebra $\mathscr{A}_{n}$ introduced and studied by

Partially supported by the Fund for the Promotion of Research at the Technion.

(C) 2004 Australian Mathematical Society 1446-7887/04 \$A2.00+0.00 
Popescu ([14] and [15]). The algebra $H^{\infty}(C)$ in this case was studied by Popescu ([14]) who denoted it $F_{n}^{\infty}$ and by Davidson and Pitts ([6]) who wrote $\mathscr{L}_{n}$ for it and referred to it as a free semigroup algebra. We shall use the notation $\mathscr{A}_{n}$ and $\mathscr{L}_{n}$ for these special quiver algebras. For more general (countable) graphs the algebra that we write as $H^{\infty}(C)$ was recently studied by Kribs and Power ([8]). They denoted it $\mathscr{L}_{G}$ where $G$ is the graph (= quiver) and called it a free semigroupoid algebra.

In [15] Popescu proved that, if $n \neq m$ then the algebras $\mathscr{A}_{n}$ and $\mathscr{A}_{m}$ are not isomorphic. One can also show that, in this case, there is no isomorphism from $\mathscr{L}_{n}$ onto $\mathscr{L}_{m}$ preserving the weak topologies (that can be deduced from [6, Theorem 2.3]). For the algebras $\mathscr{T}_{+}(C)$ and $H^{\infty}(C)$ it is shown in [8, Corollary 10.5 and Theorem 10.1] that, if the graphs are not isomorphic then the algebras cannot be unitarily equivalent. (With respect to 'regular representations' of the algebras).

The purpose of this note is to relax the condition of unitarily equivalent and replace it with isometrically isomorphic (for $\mathscr{T}_{+}(C)$ ) and isometrically isomorphic via a $w^{*}-w^{*}$-homeomorphism (for $H^{\infty}(C)$ ). This is proved in Theorem 3.7 which is the main result. Moreover, we show how to 'read' the quiver (that is, the numbers $C_{i j}$ ) from the characters and certain representations of the algebra (justifying the title of this paper).

All the graphs in this paper will be assumed to be countable. To simplify the arguments we prove the main result for graphs with finitely many vertices (that is, $n<\infty$ ) but one can extend the arguments (with some care) to general countable graphs.

The $C^{*}$-analogue of quiver algebras is referred to as graph $C^{*}$-algebras and these have been studied extensively starting with the work of Cuntz and Krieger [5]. (See also $[4,7,13,9]$ and others). It is not true that, if two graph $C^{*}$-algebras are isomorphic, then the graphs are isomorphic. Somehow the non selfadjoint algebra preserves all the data while the $C^{*}$-algebra 'forgets' some. A similar phenomenon was observed by Arveson for algebras associated with dynamical systems ([2]).

\section{Preliminaries}

We begin by recalling the notion of a $W^{*}$-correspondence. For the general theory of Hilbert $C^{*}$-modules which we use, we will follow [10]. In particular, a Hilbert $C^{*}$-module will be a right Hilbert $C^{*}$-module.

Definition 2.1. Let $A$ be a von Neumann algebra and let $E$ be a (right) Hilbert $C^{*}$-module over $A$. Then $E$ is called a Hilbert $W^{*}$-module over $A$ in case it is self dual (that is, every continuous $A$-module map from $E$ to $A$ is implemented by an element of $E$ ). It is called a $W^{*}$-correspondence over $A$ if it is also endowed with the structure of a left $A$-module via a normal *-homomorphism $\varphi: A \rightarrow \mathscr{L}(E)$. (Here $\mathscr{L}(E)$ is 
the algebra of all bounded, adjointable, module maps on $E$ ).

Given a $W^{*}$-correspondence over $A$, we denote the $A$-valued inner product on $E$ by $\langle\cdot, \cdot\rangle$. The full Fock space over $E$ will be denoted by $\mathscr{F}(E)$, so

$$
\mathscr{F}(E)=A \oplus E \oplus E^{\otimes 2} \oplus \cdots
$$

(The tensor products here are internal tensor products, see [10]). The space $\mathscr{F}(E)$ is evidently a Hilbert $W^{*}$-correspondence over $A$ with left action $\varphi_{\infty}$ given by the formula $\varphi_{\infty}(a)=\operatorname{diag}\left(a, \varphi(a), \varphi^{(2)}(a), \ldots\right)$, where

$$
\varphi^{(k)}(a)\left(\xi_{1} \otimes \xi_{2} \otimes \cdots \otimes \xi_{k}\right)=\varphi(a) \xi_{1} \otimes \xi_{2} \otimes \cdots \otimes \xi_{k} .
$$

For $\xi \in E$, we write $T_{\xi}$ for the creation operator on $\mathscr{F}(E)$ :

$$
T_{\xi}\left(\xi_{1} \otimes \xi_{2} \otimes \cdots \otimes \xi_{k}\right)=\xi \otimes \xi_{1} \otimes \xi_{2} \otimes \cdots \otimes \xi_{k} .
$$

Then $T_{\xi}$ is a continuous, adjointable operator in $\mathscr{L}(\mathscr{F}(E))$. The norm closed subalgebra of $\mathscr{L}(\mathscr{F}(E))$ generated by all the $T_{\xi}$ 's and $\varphi_{\infty}(A)$ is called the tensor algebra over $E$ and is denoted $\mathscr{T}_{+}(E)([12])$. Since $\mathscr{F}(E)$ is a Hilbert $W^{*}$-module, it is known that $\mathscr{L}(\mathscr{F}(E))$ is a von Neumann algebra. We can now close $\mathscr{T}_{+}(E)$ in the $w^{*}$-topology. This $w^{*}$-closed algebra will be denoted $H^{\infty}(E)$ and will be referred to as the weak tensor algebra of $E$.

We will be interested in a certain class of $W^{*}$-correspondences. First, let the algebra $A$ be the algebra $D_{n}$ of all diagonal $n \times n$ (complex) matrices (if $n=\infty$ let $A=l_{\infty}$ ). For a fixed $n$ let $C$ be a fixed $n \times n$ matrix with entries in $\mathbb{Z}_{+} \cup\{\infty\}$. For each $1 \leq i, j \leq n$, let $E(C)_{i j}$ be a (complex) $C_{i j}$-dimensional Hilbert space. (We will usually write it as $\mathbb{C}^{C_{i j}}$, where $\mathbb{C}^{\infty}$ is, of course, $l_{2}$ and $\mathbb{C}^{0}$ will be understood as $\{0\}$.) The space $E=E(C)$ is the vector space of all $n \times n$ matrices $\xi$ with the property that its $i, j$ entry, $\xi_{i j}$, is a vector in $E(C)_{i j}$. (If $n=\infty$, we shall also require that $\sup _{j} \sum_{i=1}^{\infty}\left\|\xi_{i j}\right\|^{2}<\infty$ holds.) This space can be viewed as an $A-A$ bimodule via the formulae:

$$
(\xi D)_{i j}=(\xi)_{i j} d_{j}, \quad(\varphi(D) \xi)_{i j}=d_{i}(\xi)_{i j}
$$

where $D=\operatorname{diag}\left(d_{1}, d_{2}, \ldots\right)$ lies in $A$. Also, $E(C)$ has an $A$-valued inner product defined by the formula $(\langle\xi, \eta\rangle)_{j}=\sum_{i=1}^{n}\left\langle\xi_{i j}, \eta_{i j}\right\rangle$. (Note that, since the $A$-valued inner product on $E(C)$ is linear in the second term, we shall use this convention also for the inner products of the Hilbert spaces $E(C)_{i j}$.)

This makes $E(C)$ a $W^{*}$-correspondence over $A$. Given $E(C)$ as above, we shall write $\mathscr{T}_{+}(C)$ for $\mathscr{T}_{+}(E(C))$ and $H^{\infty}(C)$ for $H^{\infty}(E(C))$. Note that, when $C$ is the $1 \times 1$ matrix whose entry is $n$, we can write $E(C)=\mathbb{C}^{n}$. In this case the algebra $\mathscr{T}_{+}(C)$ 
is the algebra $\mathscr{A}_{n}$ studied by Popescu in [14] and in [15], and the algebra $H^{\infty}(C)$ is the algebra $\mathscr{L}_{n}$ studied by Davidson and Pitts ([6]) and by Popescu in [14]. (Popescu denoted it by $F_{n}^{\infty}$.) For a general matrix $C$, the algebra $H^{\infty}(C)$ was studied recently by Kribs and Power ([8]). They called it a free semigroupoid algebra and denoted it by $\mathscr{L}_{G}$ where $G$ is the (countable) graph associated with $C$. They also studied norm closed algebras $\mathscr{A}_{G}\left(\left[8\right.\right.$, Corollary 10.5]). These are tensor algebras $\mathscr{T}_{+}(E(C))$ but, if $n=\infty$, one has to define the correspondence $E(C)$ as a $C^{*}$-correspondence over the $C^{*}$-algebra $c$ (or $c_{0}$ ), not over $l_{\infty}$.

The representation theory for the tensor algebras was worked out in [12]. We now describe some of the basic results.

DEFINITION 2.2. Let $E$ be a $W^{*}$-correspondence over $A$ and let $H$ be a Hilbert space.

(1) A covariant representation of $E$ on $H$ is a pair $(T, \sigma)$, where

(i) $\sigma$ is a representation of $A$ in $B(H)$;

(ii) $T: E \rightarrow B(H)$ is a linear contraction;

(iii) $T$ is a bimodule map in the sense that $T(\varphi(a) \xi b)=\sigma(a) T(\xi) \sigma(b)$ for $\xi \in E$, $a, b \in A$.

(2) We say that the covariant representation is completely contractive if $T$ is.

(3) We say that the covariant representation is normal if $\sigma$ is a normal representation and if $T$ is continuous with respect to the $\sigma$-topology of [3] on $E$ and the $\sigma$-weak topology on $B(H)$.

(4) We say that the covariant representation is isometric if, for all $\xi, \eta$ in $E$, $T(\xi)^{*} T(\eta)=\sigma(\langle\xi, \eta\rangle)$.

Given a covariant representation $(T, \sigma)$ of $E$ on $H$, we can define a linear map $\tilde{T}$ from the algebraic tensor product $E \odot_{\sigma} H$ to $H$ defined by $\tilde{T}(\xi \otimes h)=T(\xi) h$.

We have the following (see [12, Lemma 3.5 and Theorem 3.10] for the proof):

PROPOSITION 2.3. (1) If $\rho$ is a contractive representation of $\mathscr{T}_{+}(E)$ on $H$ then, setting $\sigma(a)=\rho\left(\varphi_{\infty}(a)\right)$ for $a \in A$ and $T(\xi)=\rho\left(T_{\xi}\right)$ for $\xi \in E$, the pair $(T, \sigma)$ is a covariant representation of $E$.

(2) The map $\tilde{T}$ defined above is bounded if and only if $T$ is completely bounded. In fact, $\|\tilde{T}\|=\|T\|_{c b}$. So that $\tilde{T}$ is a contraction if and only if $(T, \sigma)$ is completely contractive. In this case we view $\tilde{T}$ as a map on the completion $E \otimes_{\sigma} H$.

(3) If $(T, \sigma)$ is completely contractive then the converse to part (1) also holds, that is, there is a completely contractive representation $\rho=T \times \sigma$ of $\mathscr{T}_{+}(E)$ on $H$ such that $\sigma(a)=\rho\left(\varphi_{\infty}(a)\right)$ for $a \in A$ and $T(\xi)=\rho\left(T_{\xi}\right)$ for $\xi \in E$.

In addition to the map $\tilde{T}$ we also define the maps $\tilde{T}_{k}: E^{\otimes k} \otimes H \rightarrow H$ by $\tilde{T}_{k}\left(\xi_{1} \otimes \cdots \otimes \xi_{k}\right)=T\left(\xi_{1}\right) \cdots T\left(\xi_{k}\right) h$ and then we have $\tilde{T}_{k+1}=\tilde{T}\left(I_{E} \otimes \tilde{T}_{k}\right)$. 
Given a representation $\pi_{0}$ of $A$ on a Hilbert space $H_{0}$ we can form the Hilbert space $\mathscr{F}(E) \otimes_{\pi_{0}} H_{0}$ (where the inner product is given by $\langle X \otimes h, Y \otimes g\rangle=\left\langle h, \pi_{0}(\langle X, Y\rangle) g\right\rangle$ for $X, Y$ in $\mathscr{F}(E)$ and $h, g \in H_{0}$ ) and define an isometric covariant representation $(V, \pi)$ of $E$ on this Hilbert space by $V(\xi)=T_{\xi} \otimes I_{H_{0}}$ and $\pi(a)=\varphi_{\infty}(a) \otimes I_{H_{0}}$. Such a representation is said to be induced.

The resulting representation $V \times \pi$ of $\mathscr{T}_{+}(E)$ is, in fact, the restriction to $\mathscr{T}_{+}(E)$ of the representation induced by $\pi_{0}$ of $\mathscr{L}(\mathscr{F}(E))$ on this Hilbert space given by the formula

$$
\pi_{0}^{\mathscr{F}_{(E)}}(T)=T \otimes I_{H_{0}}, \quad T \in \mathscr{L}(\mathscr{F}(E))
$$

This shows that, when $(V, \pi)$ is a normal induced representation of $E$, the representation $V \times \pi$ can be extended to a $w^{*}$-continuous representation of $H^{\infty}(E)$. In [11, Proposition 2.8] it is shown that, if $(T, \sigma)$ is a normal completely contractive representation of $E$ satisfying $\tilde{T}_{k} \tilde{T}_{k}^{*} \rightarrow 0$ in the strong operator topology, then the minimal isometric dilation $(V, \pi)$ of $(T, \sigma)$ is an induced representation (and, thus, the associated representation $V \times \pi$ of $\mathscr{T}_{+}(E)$ can be extended to a $w^{*}$-continuous representation of $H^{\infty}(E)$ ). Since $T \times \sigma$ is then a compression of $V \times \pi$, if follows that we can also extend this representation to a $w^{*}$-continuous representation of $H^{\infty}(E)$. We summarize this discussion as follows.

LEMMA 2.4. If $(T, \sigma)$ is a normal completely contractive covariant representation of $E$ such that $\tilde{T}_{k} \tilde{T}_{k}^{*} \rightarrow 0$ in the strong operator topology, then the representation $T \times \sigma$ can be extended to a $w^{*}$-continuous representation of $H^{\infty}(E)$.

Restricting to the case $E=\mathbb{C}^{n}$ (and $H^{\infty}(E)=\mathscr{L}_{n}$ ), we have the following.

LEMMA 2.5. Suppose $V=\left(V_{1}, V_{2}, \ldots, V_{n}\right)$ is an $n$-tuple of isometries in $B(H)$ (where we allow $n=\infty$ ) whose ranges are orthogonal and the sum of the ranges is not all of $H$. Let $\rho$ be the representation of $\mathscr{T}_{+}\left(\mathbb{C}^{n}\right)\left(=\mathscr{A}_{n}\right)$ defined by $V$. Then the following hold:

(1) There is a Hilbert space $K$ and a unitary operator $v: H \rightarrow \mathscr{F}\left(\mathbb{C}^{n}\right) \otimes K$ such that $v V_{i} v^{*}=T_{e_{i}} \otimes I_{K}\left(=\pi_{0}^{F\left(C^{n}\right)}\left(T_{e_{i}}\right)\right)$, where $\left\{e_{i}\right\}$ is the standard orthonormal basis of $\mathbb{C}^{n}$ and $\pi_{0}$ is the obvious representation of $A=\mathbb{C}$ on $K$.

(2) $v \rho(\cdot) v^{*}$ is the restriction of $\pi_{0}^{\mathscr{F}\left(\mathbb{C}^{\prime \prime}\right)}$ to $\mathscr{T}_{+}\left(\mathbb{C}^{n}\right)\left(=\mathscr{A}_{n}\right)$.

(3) $\rho$ can be extended to a completely isometric isomorphism of $\mathscr{L}_{n}$ into $B(H)$ that is a $w^{*}-w^{*}$-homeomorphism onto its image.

ProOF. In [14], $n$-tuples as above were called orthogonal shifts and part (1) follows from Theorem 1.2 there. One can also deduce it from the above discussion since $(V, \pi)$ is an induced representation of $E=\mathbb{C}^{n}$ (where $\pi$ is the obvious representation 
of $(\mathbb{C}$ on $H$ ). Part (2) follows immediately from (1) and for part (3) note that the representation $\pi_{0}^{\mathscr{F}\left(\mathbb{C}^{\prime \prime}\right)}$ is the representation that maps $S \in B\left(\mathscr{F}\left(\mathbb{C}^{n}\right)\right)$ to $S \otimes I_{K}$ in $B\left(\mathscr{F}\left(\mathbb{C}^{n}\right) \otimes K\right)$. Since this representation is completely isometric and a $w^{*}-w^{*}$ homeomorphism of the von Neumann algebra $B\left(\mathscr{F}\left(\mathbb{C}^{n}\right)\right)$ onto its image, the same holds for the restriction to $\mathscr{L}_{n}$.

\section{Isomorphic quiver algebras}

In this section we prove the main results. We now fix $n<\infty$ and an $n \times n$ matrix $C$ with entries in $\mathbb{Z}_{+} \cup\{\infty\}$. Let $A$ be the $C^{*}$-algebra $D_{n}$ of all diagonal $n \times n$ matrices and let $E(C)$ be the $A-A$-correspondence defined by $C$.

We start by identifying the characters of $\mathscr{T}_{+}(C)$ and of $H^{\infty}(C)$. In order to do it we shall first embed $\mathscr{A}_{C_{i i}}$ in $\mathscr{T}_{+}(C)$ and $\mathscr{L}_{C_{i i}}$ in $H^{\infty}(C)$.

Write $\pi$ for the usual representation of $A$ on $\mathbb{C}^{n}$ and write $H$ for $\mathbb{C}^{n}$. Let $K(C)$ denote the representation space of the representation $\pi^{\mathscr{F}(E(C))}$, induced by $\pi$, that is,

$$
K(C)=\mathscr{F}(E(C)) \otimes_{\pi} H .
$$

For every $1 \leq i \leq n$ with $C_{i i} \neq 0$, write $P_{i}$ for the projection $\varphi_{\infty}\left(e_{i}\right) \otimes I_{H}$ in $B(K(C))$ and $K_{i}(C)$ for its range. Hence

$$
K_{i}(C)=\varphi_{\infty}\left(e_{i}\right)\left(\sum_{k=0}^{\infty} \oplus E(C)^{\otimes k} \otimes H\right) .
$$

For every $1 \leq i \leq n$, let $\left\{e_{i i}^{(j)}: 1 \leq j \leq C_{i i}\right\}$ be an orthonormal basis for $E(C)_{i i}$ and view these vectors as elements of $E(C)$. For $1 \leq j \leq C_{i i}$, let $V_{j}$ be the operator on $K_{i}(C)$ defined by

$$
V_{j}=\pi^{\mathscr{F}(E(C))}\left(e_{i i}^{(j)}\right) \mid K_{i}(C)
$$

(Note that $\pi^{F(E(C))}\left(e_{i i}^{(j)}\right)$ vanishes on the orthogonal complement of $K_{i}(C)$.) We get $C_{i i}$ isometries on $K_{i}(C)$ satisfying the conditions of Lemma 2.5. Letting $\Psi_{i}$ be the map $\rho$ of that lemma (with $H=K_{i}(C)$ ) composed with the embedding of $B\left(K_{i}(C)\right.$ ) into $B(K(C)$ ) (by defining the operator to be zero on the orthogonal complement of $K_{i}(C)$ ), we get the following. (Note that $H^{\infty}(C)$ can be identified with its image

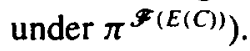

PROPOSITION 3.1. For every $1 \leq i \leq n$ with $C_{i i} \neq 0$, there is a (non unital) completely isometric isomorphism $\Psi_{i}$ of $\mathscr{L}_{C_{i i}}$ into $H^{\infty}(C)$ that is a $w^{*}$-homeomorphism (onto its image) and that restricts to a completely isometric isomorphism of $\mathscr{A}_{C_{i}}$ into $\mathscr{T}_{+}(E(C))$ (denoted also $\left.\Psi_{i}\right)$. 
A character of $\mathscr{T}_{+}(E(C))$ is a one dimensional (completely) contractive representation and, as such, it is given by a completely contractive covariant representation $(T, \tau)$ of the $W^{*}$-correspondence $E(C)$. Here $\tau$ is a one dimensional representation of $A=D_{n}$ and, thus, is $\delta_{i}$ for some $1 \leq i \leq n$ (where $\delta_{i}$ of a diagonal matrix $D=\operatorname{diag}\left(d_{1}, d_{2}, \ldots, d_{n}\right)$ is $\left.d_{i}\right)$. The map $T$ is a contractive linear functional on $E(C)$ satisfying $T\left(D_{1} \xi D_{2}\right)=\delta_{i}\left(D_{1}\right) T(\xi) \delta_{i}\left(D_{2}\right)$. Hence it is in fact a contractive linear functional on the $C_{i i}$-dimensional Hilbert space $E(C)_{i i}$. If $C_{i i}=0$ then $T=0$. Otherwise, identifying $E(C)_{i i}$ with $\mathbb{C}^{C_{i i}}$, we associate with every such character a pair $(i, \lambda)$ where $1 \leq i \leq n$ and $\lambda$ is in the closed unit ball $\overline{\mathbb{B}_{C_{i i}}}$ of $\mathbb{C}^{C_{i i}}$. If this character can be extended to a $w^{*}$-continuous character of $H^{\infty}(C)$ then, using the map $\Psi_{i}$ of Proposition 3.1, it induces a $w^{*}$-continuous character on $\mathscr{L}_{\mathrm{C}_{\mathrm{ii}}}$. It follows from Theorem 2.3 of [6] that $\lambda$ lies in the open unit ball. We summarize the discussion in the following theorem. To simplify the statement, we shall assume that, whenever $C_{i i}=0$, the notation $\mathbb{B}_{C_{i i}}$ (or its closure) will be interpreted as $\{0\}$. When $C_{i i}=\infty$ the balls $\mathbb{B}_{C_{i i}}$ and $\overline{\mathbb{B}_{C_{i i}}}$ are the balls of $l^{2}$ equipped with the weak topology.

THEOREM 3.2. Let $M\left(\mathscr{T}_{+}(C)\right)$ be the set of all characters of the quiver algebra $\mathscr{T}_{+}(C)$ equipped with the $w^{*}$-topology and let $M\left(H^{\infty}(C)\right)$ be the set of all $w^{*}-$ continuous characters of $H^{\infty}(C)$ (also with the $w^{*}$-topology).

Then we have the following homeomorphisms:

(1) $M\left(\mathscr{T}_{+}(C)\right) \cong \bigcup\left\{(i, \lambda): \lambda \in \overline{\mathbb{B}_{C_{i i}}}, 1 \leq i \leq n\right\}$.

(2) $M\left(H^{\infty}(C)\right) \cong \bigcup\left\{(i, \lambda): \lambda \in \mathbb{B}_{C_{i i}}, 1 \leq i \leq n\right\}$.

(Each set on the right hand side is a disjoint union of $n$ closed and open sets.) The character, $\varphi_{(i, \lambda)}$, associated with $(i, \lambda)$ is equal $\delta_{i}$ on $A$ and on $T_{\xi}$, for $\xi \in E(C)$, it is defined by $\varphi_{(i, \lambda)}\left(T_{\xi}\right)=\left\langle\lambda, \xi_{i i}\right\rangle$.

ProOF. The identifications in both (1) and (2) were shown above. The fact that these are homeomorphisms is easy to check.

In the following we shall also interpret $\mathbb{C}^{m}$ and $\mathbb{B}_{m}$ as $\{0\}$ if $m=0$. Now fix $1 \leq i, j \leq n, i \neq j$ and $\tilde{\lambda}=\left(\lambda_{i}, \lambda_{j}\right) \in \mathbb{B}_{C_{i i}} \times \mathbb{B}_{C_{j j}}$ and define $G(C, \tilde{\lambda}, i, j)$ (respectively, $G_{0}(C, \tilde{\lambda}, i, j)$ ) to be the set of all contractive representations $\rho$ of $\mathscr{T}_{+}(C)$ (respectively, all contractive $w^{*}$-continuous representations of $H^{\infty}(C)$ ) on the space $\mathbb{C}^{2}$ satisfying:

(G1) For $D \in A, \rho(D)=\operatorname{diag}\left(\delta_{i}(D), \delta_{j}(D)\right)$.

(G2) The image of $\rho$ is contained in $T_{2}$, the upper triangular $2 \times 2$ matrices.

(G3) For every $S$ in the algebra, $(\rho(S))_{11}=\varphi_{\left(i, \lambda_{i}\right)}(S)$ and $(\rho(S))_{22}=\varphi_{\left(j, \lambda_{j}\right)}(S)$.

We now present examples of representations in $G(C, \tilde{\lambda}, i, j)$. Write $\sigma$ for the representation of $A$ on $\mathbb{C}^{2}$ given by $\sigma(D)=\operatorname{diag}\left(\delta_{i}(D), \delta_{j}(D)\right)$. For $\gamma$ in $\mathbb{C}^{C_{i j}}$, define 
the map $T_{\gamma}: E(C) \rightarrow T_{2}$ by

$$
T_{\gamma}(\xi)=\left(\begin{array}{cc}
\varphi_{\left(i, \lambda_{i}\right)}\left(\xi_{i i}\right) & \left\langle\gamma, \xi_{i j}\right\rangle \\
0 & \varphi_{\left(j, \lambda_{j}\right)}\left(\xi_{j j}\right)
\end{array}\right)=\left(\begin{array}{cc}
\left\langle\lambda_{i}, \xi_{i i}\right\rangle & \left\langle\gamma, \xi_{i j}\right\rangle \\
0 & \left\langle\lambda_{j}, \xi_{j j}\right\rangle
\end{array}\right) \in T_{2}
$$

Then the pair $\left(T_{\gamma}, \sigma\right)$ satisfies $T_{\gamma}\left(D_{1} \xi D_{2}\right)=\sigma\left(D_{1}\right) T_{\gamma}(\xi) \sigma\left(D_{2}\right)$ for all $D_{1}, D_{2}$ in $A$ and $\xi$ in $E(C)$.

Before we proceed to show that this construction gives us a representation in $G(C, \tilde{\lambda}, i, j)$ we observe that the converse holds.

LEMMA 3.3. Let $\rho$ be a representation in $G(C, \tilde{\lambda}, i, j)$ and let $T(\xi)$ be $\rho\left(T_{\xi}\right)$ (in $\left.T_{2}\right)$. Then there is some $\gamma$ in $\mathbb{C}^{C_{i j}}$ with $\|\gamma\|^{2}+\left\|\lambda_{i}\right\|^{2} \leq 1$ such that $T=T_{\gamma}$.

Proof. Since $\rho \in G(C, \tilde{\lambda}, i, j)$, it follows that $T(\xi)_{11}=\varphi_{\left(i, \lambda_{i}\right)}\left(T_{\xi}\right)=\left\langle\lambda_{i}, \xi_{i i}\right\rangle$, $T(\xi)_{22}=\left\langle\lambda_{j}, \xi_{j j}\right\rangle$ and, for $D \in A, \rho\left(\varphi_{\infty}(D)\right)=\sigma(D)$. Hence $T\left(D_{1} \xi D_{2}\right)=$ $\sigma\left(D_{1}\right) T(\xi) \sigma\left(D_{2}\right)$ (for $D_{i}$ in $A$ ) and it follows that $T(\xi)_{12}$ depends only on $\xi_{i j}$. Since this dependence is clearly linear and $T$ is bounded, we find that $T(\xi)_{12}$ is $\left\langle\gamma, \xi_{i j}\right\rangle$ for some $\gamma \in \mathbb{C}^{C_{i}}$. Thus $T=T_{\gamma}$. To show that $\|\gamma\|^{2}+\left\|\lambda_{i}\right\|^{2} \leq 1$ let $\xi \in E(C)$ be defined by : $\xi_{i i}=\lambda_{i} /\left\|\lambda_{i}\right\|, \xi_{i j}=\gamma /\|\gamma\|$ and $\xi_{l p}=0$ otherwise. (The case where either $\gamma$ or $\lambda_{i}$ is zero can be handled easily.)

Then $\langle\xi, \xi\rangle$ is the diagonal matrix whose $i$ th diagonal entry is $\left\|\xi_{i i}\right\|^{2}=1$, the $j$ th diagonal entry is $\left\|\xi_{i j}\right\|^{2}=1$ and all other entries equal zero.

Hence $\left\|T_{\xi}\right\|=\|\xi\|=1$ and, consequently, $\left\|T_{\gamma}(\xi)\right\|=\left\|\rho\left(T_{\xi}\right)\right\| \leq 1$. But

$$
T_{\gamma}(\xi)=\left(\begin{array}{cc}
\left\langle\lambda_{i}, \xi_{i i}\right\rangle & \left\langle\gamma, \xi_{i j}\right\rangle \\
0 & 0
\end{array}\right)=\left(\begin{array}{cc}
\left\|\lambda_{i}\right\| & \|\gamma\| \\
0 & 0
\end{array}\right)
$$

Thus $1 \geq\left\|T_{\gamma}(\xi)\right\|^{2}=\left\|\lambda_{i}\right\|^{2}+\|\gamma\|^{2}$.

Now recall that the pair $\left(T_{\gamma}, \sigma\right)$ defines a map $\tilde{T}_{\gamma}$ from the algebraic tensor product $E(C) \odot_{\sigma} \mathbb{C}^{2}$ to $\mathbb{C}^{2}$ satisfying $\tilde{T}_{\gamma}(\xi \otimes h)=T_{\gamma}(\xi) h$ which is bounded (respectively, contractive) if and only if $T_{\gamma}$ is completely bounded (respectively, completely contractive). If $\tilde{T}_{\gamma}$ is contractive, then (using Proposition 2.3) the pair $\left(T_{\gamma}, \sigma\right)$ defines a completely contractive representation $T_{\gamma} \times \sigma$ of $\mathscr{T}_{+}(C)$.

The proof of the following lemma is a straightforward computation and is omitted.

LEMMA 3.4. Let $T_{\gamma}$ be as above (for some $\gamma \in \mathbb{C}^{C_{i j}}$ ) and fix $\xi$ in $E(C)$. Then, for $k=\left(k_{1}, k_{2}\right)^{t}$ and $h=\left(h_{1}, h_{2}\right)^{t}$ in $\mathbb{C}^{2}$ we have

(1) $\tilde{T}_{\gamma}(\xi \otimes h)=\left(\begin{array}{c}\left\langle\lambda_{i}, \xi_{i i}\right\rangle h_{1}+\left\langle\gamma, \xi_{i j}\right\rangle h_{2} \\ \left\langle\lambda_{j}, \xi_{j j}\right\rangle h_{2}\end{array}\right)$.

(2) $\tilde{T}_{\gamma}^{*} k=\eta \otimes\left(\begin{array}{l}1 \\ 1\end{array}\right)$, where $\eta$ is the element of $E(C)$ with $\eta_{i i}=k_{1} \lambda_{i}, \eta_{i j}=k_{1} \gamma$, $\eta_{j j}=k_{2} \lambda_{j}$ and all other entries are 0 . 
(3) $\tilde{T}_{\gamma} \tilde{T}_{\gamma}^{*}=\left(\begin{array}{cc}\left\|\lambda_{i}\right\|^{2}+\|\gamma\|^{2} & 0 \\ 0 & \left\|\lambda_{j}\right\|^{2}\end{array}\right)$.

In part (2) of the following corollary we present the general form of the representations in $G(C, \tilde{\lambda}, i, j)$.

COROLLARY 3.5. (1) $\left\|\tilde{T}_{\gamma}\right\| \leq 1$ (that is, it defines a completely contractive representation $\rho_{\gamma}=T_{\gamma} \times \sigma$ of the algebra $\mathscr{T}_{+}(C)$ ) if and only if $\|\gamma\|^{2} \leq 1-\left\|\lambda_{i}\right\|^{2}$.

(2) The representations in $G(C, \tilde{\lambda}, i, j)$ are all completely contractive and of the form $\rho_{y}=T_{\gamma} \times$ o (for some $\gamma$ in $\mathbb{C}^{C_{i j}}$ with $\|\gamma\|^{2} \leq 1-\left\|\lambda_{i}\right\|^{2}$ ).

(3) When $\|\gamma\|^{2} \leq 1-\left\|\lambda_{i}\right\|^{2}$, we have $\left\|\tilde{T}_{\gamma, k}\right\| \rightarrow 0$. Hence $\tilde{T}_{\gamma}$ defines a $w^{*}$-continuous representation of $H^{\infty}(C)$. Therefore, $G(C, \tilde{\lambda}, i, j)=G_{0}(C, \tilde{\lambda}, i, j)$.

PROOF. Parts (1) and (2) follow from Lemma 3.4 and Lemma 3.3. For part (3), fix $\gamma$ with $\|\gamma\|^{2} \leq 1-\left\|\lambda_{i}\right\|^{2}$ and write $T$ for $T_{\gamma}$. Recall that $\tilde{T}_{k}$ is a map from $E^{\otimes k} \otimes \mathbb{C}^{2}$ to $\mathbb{C}^{2}$ defined recursively by $\tilde{T}_{1}=\tilde{T}$ and $\tilde{T}_{k+1}=\tilde{T}\left(I_{E} \otimes \tilde{T}_{k}\right)$. Hence $\tilde{T}_{k+1} \tilde{T}_{k+1}^{*}=\tilde{T}\left(I \otimes \tilde{T} \tilde{T}^{*}\right) \tilde{T}^{*}$. Thus, if $\tilde{T}_{k} \tilde{T}_{k}^{*}=\left(\begin{array}{cc}a & 0 \\ 0 & b\end{array}\right)$ then, for $g=\left(g_{1}, g_{2}\right)^{t}$ in $\mathbb{C}^{2}$,

$$
\tilde{T}_{k+1} \tilde{T}_{k+1}^{*} g=\tilde{T}\left(I \otimes\left(\begin{array}{ll}
a & 0 \\
0 & b
\end{array}\right)\right) \tilde{T}^{*} g=\tilde{T}\left(\eta \otimes\left(\begin{array}{l}
a \\
b
\end{array}\right)\right)
$$

where $\eta_{i i}=g_{1} \lambda_{i}, \eta_{i j}=g_{2} \gamma, \eta_{j j}=g_{2} \lambda_{j}$ and $\eta_{l p}=0$ otherwise. Hence

$$
\begin{aligned}
\tilde{T}_{k+1} \tilde{T}_{k+1}^{*} g & =\left(\begin{array}{c}
\left\langle\lambda_{i}, g_{1} \lambda_{i}\right\rangle a+\left\langle\gamma, g_{1} \gamma\right\rangle b \\
\left\langle\lambda_{j}, g_{2} \lambda_{j}\right\rangle b
\end{array}\right)=\left(\begin{array}{c}
g_{1} a\left\|\lambda_{i}\right\|^{2}+g_{1} b\|\gamma\|^{2} \\
g_{2} b\left\|\lambda_{j}\right\|^{2}
\end{array}\right) \\
& =\left(\begin{array}{cc}
a\left\|\lambda_{i}\right\|^{2}+b\|\gamma\|^{2} & 0 \\
0 & b\left\|\lambda_{j}\right\|^{2}
\end{array}\right) g
\end{aligned}
$$

and

$$
\tilde{T}_{k+1} \tilde{T}_{k+1}^{*}=\left(\begin{array}{cc}
a\left\|\lambda_{i}\right\|^{2}+b\|\gamma\|^{2} & 0 \\
0 & b\left\|\lambda_{j}\right\|^{2}
\end{array}\right)
$$

Write $q_{i}=\left\|\lambda_{i}\right\|^{2}$ and $t=\|\gamma\|^{2}$. Then the computation above shows that

$$
\|\tilde{T}\|^{2}=\max \left\{q_{1}+t, q_{2}\right\}, \quad\left\|\tilde{T}_{2}\right\|^{2}=\max \left\{q_{1}^{2}+t q_{1}+t q_{2}, q_{2}^{2}\right\}
$$

and, in general,

$$
\left\|\tilde{T}_{k}\right\|^{2}=\max \left\{q_{1}^{k}+t\left(q_{1}^{k-1}+q_{1}^{k-2} q_{2}+\cdots+q_{2}^{k-1}\right), q_{2}^{k}\right\}
$$

If $q=\max \left\{q_{1}, q_{2}\right\}$ then $q<1$ and $\left\|\tilde{T}_{k}\right\|^{2} \leq q^{k}+k t q^{k-1} \rightarrow 0$. Using Lemma 2.4 we see that this implies that the representation $\rho_{\gamma}$ can be extended to a $w^{*}$-continuous representation of $H^{\infty}(C)$. 
We now equip the set $G(C, \tilde{\lambda}, i, j)$ with the topology of pointwise convergence. It is then homeomorphic to a closed subset of the product space

$$
\prod\left\{\overline{\mathbb{B}}\left(T_{2}\right): S \in \mathscr{T}_{+}(C),\|S\| \leq 1\right\}
$$

(equipped with the product topology), where $\bar{B}\left(T_{2}\right)$ is the closed unit ball of $T_{2}$. This shows that, in this topology, $G(C, \tilde{\lambda}, i, j)$ is a compact set. For every $\eta$ in $E(C)_{i j}\left(\cong \mathbb{C}^{C_{i j}}\right)$,

$$
\langle\gamma, \eta\rangle=\left(T_{\gamma}\left(\begin{array}{ll}
0 & \eta \\
0 & 0
\end{array}\right)\right)_{12}=\left(\rho_{\gamma}\left(T_{\tilde{\eta}}\right)\right)_{12}
$$

where $\tilde{\eta}=\left(\begin{array}{ll}0 & \eta \\ 0 & 0\end{array}\right)$. If $\rho_{\gamma_{s}} \rightarrow \rho_{\gamma}$ in $G(C, \tilde{\lambda}, i, j)$ then, for every $\eta \in E(C)_{i j},\left\langle\gamma_{\alpha}, \eta\right\rangle \rightarrow$ $\langle\gamma, \eta\rangle$, that is, $\gamma_{\alpha} \rightarrow \gamma$ in the weak topology (of $E(C)_{i j}$ ). Since the map $\rho_{\gamma} \mapsto \gamma$ is a bijection and the spaces are compact, we conclude that it is a homeomorphism. We summarize it in the following proposition.

PROPOSITION 3.6. The set $G(C, \tilde{\lambda}, i . j)$, equipped with the topology of pointwise convergence, is homeomorphic to a closed ball (of positive radius) in $\mathbb{C}^{C_{i j}}$ (equipped with the weak topology).

THEOREM 3.7. Let $C$ be in $M_{n}\left(\mathbb{Z}_{+} \cup\{\infty\}\right)$ and $C^{\prime}$ be in $M_{m}\left(\mathbb{Z}_{+} \cup\{\infty\}\right)$.

(1) If the algebras $\mathscr{T}_{+}(C)$ and $\mathscr{T}_{+}\left(C^{\prime}\right)$ are isometrically isomorphic then $n=m$ and there is a permutation $\tau \in S_{n}$ such that $C_{i j}^{\prime}=C_{\tau(i) \tau(j)}$ for all $i, j$.

(2) If the algebras $H^{\infty}(C)$ and $H^{\infty}\left(C^{\prime}\right)$ are isometrically isomorphic via an isomorphism that is $w^{*}$-bicontinuous then $n=m$ and there is a permutation $\tau \in S_{n}$ such that $C_{i j}^{\prime}=C_{\tau(i) \tau(j)}$ for all $i, j$.

Proof. We start by proving (1). Write $\Lambda: \mathscr{T}_{+}(C) \rightarrow \mathscr{T}_{+}\left(C^{\prime}\right)$ for the isometric isomorphism. The selfadjoint part of $\mathscr{T}_{+}(C), \mathscr{T}_{+}(C) \cap \mathscr{T}_{+}(C)^{*}$, is equal to $\varphi_{\infty}(A)$ which is isomorphic to $D_{n}$. Since $\Lambda$ is an isometry, it maps the selfadjoint part of $\mathscr{T}_{+}(C)$ onto the selfadjoint part of $\mathscr{T}_{+}\left(C^{\prime}\right)([1])$, thus inducing an isomorphism of $D_{n}$ onto $D_{m}$. This shows that $n=m$ and there is some permutation $\tau \in S_{n}$ such that, whenever $D$ is in $A$,

$$
\Lambda\left(\varphi_{\infty}(D)\right)=\varphi_{\infty}\left(\tau^{(n)}(D)\right)
$$

where $\tau^{(n)}\left(\operatorname{diag}\left(d_{1}, \ldots, d_{n}\right)\right)=\operatorname{diag}\left(\tau\left(d_{1}\right), \ldots, \tau\left(d_{n}\right)\right)$. Fix a character, $\varphi=\varphi_{(i, \lambda)}$ in $M\left(\mathscr{T}_{+}\left(C^{\prime}\right)\right)$. Then $\varphi \circ \Lambda$ is in $M\left(\mathscr{T}_{+}(C)\right)$. Restricted to $A$, the character $\varphi=\varphi_{(i, \lambda)}$ vanishes on diagonal matrices whose $i$ th entry is 0 . Thus $\varphi \circ \Lambda$ vanishes on these diagonal matrices whose $\tau^{-1}(i)$ th entry is zero. We can write

$$
\varphi_{(i, \lambda)} \circ \Lambda=\varphi_{\left(\tau^{-1}(i), \lambda^{\prime}\right)} .
$$


In fact, it is clear from Theorem 3.2 (part (1)) that $\lambda \mapsto \lambda^{\prime}$ is a homeomorphism,

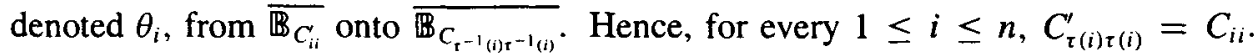
Now fix $i \neq j$. For a representation $\rho \in G\left(C^{\prime}, \mathbf{0}, i, j\right)$ (where $0=(0,0)$ ), $\rho \circ \Lambda$ is a contractive representation of $\mathscr{T}_{+}(C)$ whose image is contained in $T_{2}$. For $D=$ $\operatorname{diag}\left(d_{1}, d_{2}, \ldots d_{n}\right)$ in $A$,

$$
\rho \circ \Lambda(D)=\rho\left(\operatorname{diag}\left(\tau\left(d_{1}\right), \ldots \tau\left(d_{n}\right)\right)\right)=\operatorname{diag}\left(\delta_{\mathrm{r}(i)}(D), \delta_{\tau(j)}(D)\right) .
$$

Also, for $S \in \mathscr{T}_{+}(C)$,

$$
\rho \circ \Lambda(S)_{11}=\rho(\Lambda(S))_{11}=\varphi_{(i, 0)}(\Lambda(S))=\varphi_{\left(\tau^{-1}(i), \theta_{i}(0)\right)}(S)
$$

and, similarly,

$$
\rho \circ \Lambda(S)_{22}=\varphi_{\left(\tau^{-1}(j), \theta_{j}(0)\right)}(S) .
$$

Since $\theta_{i}$ and $\theta_{j}$ are homeomorphisms of the closed unit balls and 0 is an interior point, $\theta_{i}(0)$ and $\theta_{j}(0)$ are in the open unit balls. We write $\tilde{\lambda}=\left(\theta_{i}(0), \theta_{j}(0)\right)$ and conclude that $\rho \circ \Lambda$ lies in $G\left(C, \tilde{\lambda}, \tau^{-1}(i), \tau^{-1}(j)\right)$.

Since the map $\rho \mapsto \rho \circ \Lambda$ is a homeomorphism (with respect to the topology of pointwise convergence) we get (using Proposition 3.6) a homeomorphism of the

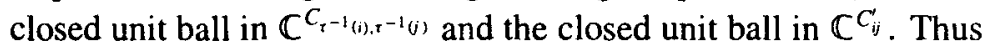

$$
C_{\tau^{-1}(i), \tau^{-1}(j)}=C_{i j}^{\prime} .
$$

This proves part (1). The proof of part (2) is almost identical except that in the argument showing $C_{i i}=C_{\tau(i) \tau(i)}^{\prime}$ we use open balls (and part (2) of Theorem 3.2) instead of closed balls. The proof for $i \neq j$ is the same due to the fact that every representation in $G(C, \tilde{\lambda}, i, j)$ extends to a $w^{*}$-continuous representation of $H^{\infty}(C)$ (Corollary 3.5 (3)).

\section{References}

[1] J. Arazy and B. Solel, 'Isometries of non-self-adjoint operator algebras', J. Funct. Anal. 90 (1990), 284-305.

[2] W. B. Arveson, 'Operator algebras and measure preserving automorphisms', Acta Math. 118 (1967), 95-109.

[3] M. Baillet, Y. Denizeau and J.-F. Havet, 'Indice d'une esperance conditionnelle', Comp. Math. 66 (1988), 199-236.

[4] B. Brenken, ' $C *$-algebras of infinite graphs and Cuntz-Krieger algebras', Canad. Math. Bull. 45 (2002), 321-336. 
[5] J. Cuntz and W. Krieger, 'A class of $C^{*}$-algebras and topological Markov chains', Invent. Math. 56 (1980), 251-268.

[6] K. R. Davidson and D. R. Pitts, 'The algebraic structure of noncommutative analytic Toeplitz algebras', Math. Ann. 311 (1998), 275-303.

[7] R. Exel and M. Laca, 'Cuntz-Krieger algebras for infinite matrices', J. Reine Angew. Math. 512 (1999), 119-172.

[8] D. W. Kribs and S. C. Power, 'Free semigroupoid algebras', preprint.

[9] A. Kumjian, D. Pask and I. Raeburn, 'Cuntz-Krieger algebras of directed graphs', Pacific J. Math. 184 (1998), 161-174.

[10] E. C. Lance, Hilbert $C^{*}$-modules-a toolkit for operator algebraists, London Math. Soc. Lecture Notes (Cambridge Univ. Press, Cambridge, UK, 1995).

[11] P. S. Muhly and B. Solel, 'The curvature and index of completely positive maps', Proc. London Math. Soc. (3) 87 (2003), 748-778.

[12] _ 'Tensor algebras over $C^{*}$-correspondences (representations, dilations and $C^{*}$-envelopes)', J. Funct. Anal. 158 (1998), 389-457.

[13] M. Laca N. Fowler and I. Raeburn, 'The $C^{*}$-algebras of infinite graphs', Proc. Amer. Math. Soc. 128 (2000), 2319-2327.

[14] G. Popescu, 'von Neumann inequality for $(B(\mathscr{H}))_{1}$ ', Math. Scand. 68 (1991), 292-304.

[15] — , 'Non commutative disc algebras and their representations', Proc. Amer. Math. Soc. 124 (1996), 2137-2148.

Department of Mathematics

Technion

Haifa 32000

Israel

e-mail: mabaruch@techunix.technion.ac.il 